\title{
NEW COMPUTATIONAL APPROACH FOR THE DESIGN OF FAULT DETECTION AND ISOLATION FILTERS
}

\author{
A. Varga \\ German Aerospace Center \\ DLR - Oberpfaffenhofen \\ Institute of Robotics and Mechatronics \\ D-82234 Wessling, Germany. \\ E-mail: Andras.Vargaedlr.de
}

\begin{abstract}
We propose a numerically reliable computational approach for the design of residual generators for fault detection and isolation filters. The new approach is based on computing solutions of least dynamical orders of linear equations with rational matrix coefficients in combination with special rational factorizations. The main computational ingredients are the orthogonal reduction of the associated system matrix pencil to a certain Kronecker-like staircase form, the solution of a minimal dynamic cover design problem, and the computation of stable and proper rational factorizations with diagonal denominators. For all these computations we discuss numerically reliable algorithms relying on matrix pencil and descriptor system techniques. The proposed residual generator design approach is completely general, is applicable to both continuous- and discrete-time systems, and can easily handle even unstable and/or improper systems.
\end{abstract}

Keywords: fault detection, fault isolation, linear rational equations, rational factorizations, numerical methods. 


\section{INTRODUCTION}

In the model based fault diagnosis, the fault detection task is achieved by detecting discrepancies between the outputs of the monitored plant and the predictions obtained with a mathematical model. These discrepancies - called also residuals - are indications of faults and are produced by special devices called residual generators. From a system theoretic point of view, the residual generators are physically realizable systems having as inputs the measured outputs and the control inputs of the monitored system, and as outputs the generated residuals. The residual generators are usually implemented as parts of control algorithms or as independent monitoring procedures.

Several algorithms underlying the design of residual generators require the manipulation of rational matrices. For low dimensional systems, this is possible to some extent by using symbolic manipulation software as provided by tools like Maple or Mathematica. However for large order systems, symbolic computation is not anymore applicable because of tremendous manipulation efforts, and therefore the use of numerical algorithms is the only possible option. The need to address the numerical issues encountered in designing fault detection and isolation filters has been already recognized by Chen and Patton (1999). By using recently developed numerically reliable descriptor system algorithms, many of computational problems in the fault detection field can be addressed for high dimensional systems.

We propose a new computational approach for the design of residual generators for fault detection and isolation filters based on solving linear equations with rational matrix coefficients. Additionally, rational factorization techniques are employed to ensure the properness and stability of the resulting residual generators. For the solution of these computational problems we propose numerically reliable algorithms relying on descriptor system techniques. The main computational ingredient in solving linear equations with rational matrices is the orthogonal reduction of the associated system matrix pencil to a certain Kronecker-like staircase form. Using this form a solution can be easily constructed, without the need to explicitly invert any rational or polynomial matrix. To determine stable and proper solutions of least dynamical orders, minimal dynamic cover design techniques in combination with coprime factorization procedures are employed. The proposed computational approach to design residual generators for fault detection and isolation filters is completely general, is applicable to both continuous- and discrete-time systems, and can easily handle even unstable and/or improper systems. The design procedure of residual generators can be easily implemented using the robust numerical tools available in the DESCRIPTOR SySTEMS Toolbox developed by the author ${ }^{1}$.

\footnotetext{
${ }^{1}$ http://www.robotic.dlr.de/control/num/desctool.html
} 


\section{DESIGN OF FAULT DETECTION AND ISOLATION FILTERS}

Consider the linear time-invariant system described by the input-output relations

$$
\mathbf{y}(\lambda)=G_{p}(\lambda) \mathbf{u}(\lambda)+G_{f}(\lambda) \mathbf{f}(\lambda)+G_{d}(\lambda) \mathbf{d}(\lambda),
$$

where $\mathbf{y}(\lambda), \mathbf{u}(\lambda), \mathbf{f}(\lambda)$, and $\mathbf{d}(\lambda)$ are Laplace- or Z-transformed vectors of the $p$ dimensional system output vector $y(t), m$-dimensional plant input vector $u(t), q$ dimensional fault signal vector $f(t)$, and $r$-dimensional disturbance vector $d(t)$, respectively, and where $G_{p}(\lambda), G_{f}(\lambda)$ and $G_{d}(\lambda)$ are the transfer-function matrices (TFMs) from the plant inputs to outputs, fault signals to outputs, and disturbances to outputs, respectively. According to the system type, $\lambda=s$ in the case of a continuous-time system or $\lambda=z$ in the case of a discrete-time system.

The fault detection and isolation (FDI) problem can be formulated as follows: determine a linear residual generator (or detector) of least dynamical order having the general form

$$
\mathbf{r}(\lambda)=R(\lambda)\left[\begin{array}{l}
\mathbf{y}(\lambda) \\
\mathbf{u}(\lambda)
\end{array}\right]
$$

such that: $(i) r(t)=0$ when $f(t)=0$; and $(i i) r_{i}(t) \neq 0$ when $f_{i}(t) \neq 0$, for $i=1, \ldots, q$. Besides the requirement that the TFM of the detector $R(\lambda)$ has least possible McMillan degree, it is also necessary, for physical realizability, that $R(\lambda)$ is a proper and stable TFM.

One possibility to determine a least order $R(\lambda)$ is to solve the following minimal design problem (Varga, 2002): choose a suitable diagonal $M(\lambda)$ (i.e., stable, proper and invertible) and find a least McMillan degree solution $R(\lambda)$ of the linear equation with rational matrices

$$
R(\lambda)\left[\begin{array}{ccc}
G_{f}(\lambda) & G_{d}(\lambda) & G_{p}(\lambda) \\
O & O & I_{m}
\end{array}\right]=\left[\begin{array}{ccc}
M(\lambda) & O & O
\end{array}\right]
$$

which is stable and proper. This equation arises by imposing for a detector of the general form (2) the condition that $r(\lambda)=M(\lambda) f(\lambda)$ for all $d(\lambda)$ and $u(\lambda)$.

To solve the above equation for properly chosen $M(\lambda)$, the minimum degree algorithm of Wang and Davison (1973) can be considered as basis for a possible numerical approach using polynomial techniques. Alternatively, provided $\left[G_{f}(\lambda) G_{d}(\lambda)\right]$ is left invertible, a numerically reliable inversion based procedure has been proposed by Varga (2002). Here we compute first (using a state space based approach) a least order left-inverse $G^{+}(\lambda)$ of

$$
G(\lambda)=\left[\begin{array}{ccc}
G_{f}(\lambda) & G_{d}(\lambda) & G_{p}(\lambda) \\
O & O & I_{m}
\end{array}\right]
$$


and then determine $R(\lambda)$ as the numerator of a stable and proper fractional representation $G_{1}^{+}(\lambda)=M(\lambda)^{-1} R(\lambda)$, where $G_{1}^{+}(\lambda)$ represents the first $q$ rows of $G^{+}(\lambda)$ and $M(\lambda)$ is diagonal. Note that determining a least order $R(\lambda)$ is part of the computation of the left-inverse $G^{+}(\lambda)$, and can be explicitly addressed.

In this paper we propose an alternative approach based on solving the rational equation (3) to obtain a least order stable and proper solution $R(\lambda)$ by choosing an appropriate $M(\lambda)$. This computation can be performed in several steps involving manipulation of rational matrices. For each step we propose matrix pencil based reliable numerical algorithms which allow to determine the solution by computing exclusively with real matrices of state space models. By using the proposed approach, the fault detection and isolation filter design problem can be solved in the most general setting. Thus our approach represents a completely general solution to the FDI problem, being a numerically reliable computational alternative to various inversion based methods (Ding and Frank, 1990; Frank and Ding, 1994; Gertler and Monajemy, 1995; Peng et al., 1997; Gertler, 1998; Chen and Patton, 1999; Gertler, 2000).

\section{SOLVING RATIONAL EQUATIONS}

For the design of residual generators in the most general setting, we have to solve a rational equation of the form (3), where we have the additional freedom of choosing a diagonal $M(\lambda)$ such that the resulting $R(\lambda)$ is proper and stable. Since the solution is in general non-unique, we would like to compute a solution which has the least McMillan degree.

In order to solve this problem, we consider, for convenience, the more general dual problem to solve a linear rational system of the form

$$
G(\lambda) X(\lambda)=F(\lambda) M(\lambda)
$$

where $G(\lambda)$ and $F(\lambda)$ are given $p \times m$ and $p \times q$ rational TFMs, respectively, and we need to choose an invertible diagonal $M(\lambda)$ such that the resulting solution $X(\lambda)$ is proper, stable and has the least possible McMillan degree. It is a well known fact that the system (4) has a solution provided the rank condition

$$
\operatorname{rank} G(\lambda)=\operatorname{rank}[G(\lambda) F(\lambda)]
$$

is fulfilled. We assume throughout the paper that this condition holds.

For a given $M(\lambda)$ the general solution of (4) can be expressed as

$$
\widehat{X}(\lambda)=X_{0}(\lambda)+X_{N}(\lambda) Y(\lambda)
$$


where $X_{0}(\lambda)$ is a particular solution of (4) and $X_{N}(\lambda)$ is a rational basis matrix for the right nullspace of $G(\lambda)$. Thus a straightforward procedure to solve (4) would be to compute first $X_{0}(\lambda)$ and $X_{N}(\lambda)$ for $M(\lambda)=I$, then to determine a suitable $Y(\lambda)$ to obtain a solution $\widehat{X}(\lambda)$ of least McMillan degree, and finally to choose an appropriate $M(\lambda)$ ensuring the stability and properness of $X(\lambda)=\widehat{X}(\lambda) M(\lambda)$.

The main difficulty using this approach is the computation of $Y(\lambda)$ in the case when $X_{0}(\lambda)$ is not proper. In this case the corresponding $Y(\lambda)$ can be improper as well and for this computation there is no known computational procedure. In contrast, if $X_{0}(\lambda)$ and $X_{N}(\lambda)$ are proper rational matrices, then the resulting proper $Y(\lambda)$ can be determined by employing the approach proposed by Morse (1976) based on minimal cover design techniques. The following conceptual procedure is merely a refining of the above steps in order to guarantee the applicability of the approach of Morse (1976). For this, we determine $M(\lambda)$ in a factored form $M(\lambda)=M_{f}(\lambda) M_{s}(\lambda)$, where $M_{f}(\lambda)$ is a proper and stable factor chosen to ensure the properness of $X_{0}(\lambda)$ and $M_{s}(\lambda)$ is a proper and stable factor chosen to ensure the stability of the solution $X(\lambda)$. In what follows we formalize the main steps of the solution procedure and subsequently we discuss suitable computational methods based on pencil manipulation techniques to perform these steps.

1. Compute a particular solution $X_{0}(\lambda)$ satisfying $G(\lambda) X_{0}(\lambda)=F(\lambda)$.

2. Compute a proper rational basis $X_{N}(\lambda)$ of the right nullspace of $G(\lambda)$.

3. Compute a diagonal $M_{f}(\lambda)$ having the least McMillan degree such that $\widehat{X}_{0}(\lambda):=$ $X_{0}(\lambda) M_{f}(\lambda)$ is proper.

4. Determine a proper $Y(\lambda)$, such that the solution $\widehat{X}(\lambda)=\widehat{X}_{0}(\lambda)+X_{N}(\lambda) Y(\lambda)$ has the least possible McMillan degree.

5. Determine a diagonal $M_{s}(\lambda)$ having the least McMillan degree such that $X(\lambda):=$ $\widehat{X}(\lambda) M_{s}(\lambda)$ is stable.

In what follows we discuss numerically reliable state space computational algorithms for each step of the above procedure.

\subsection{Computation of $X_{0}(\lambda)$}

Let assume that the compound TFM $[G(\lambda) F(\lambda)]$ has a minimal descriptor realization of order $n$ of the form

$$
\begin{aligned}
E \lambda x(t) & =A x(t)+B_{G} u(t)+B_{F} \nu(t) \\
\xi(t) & =C x(t)+D_{G} u(t)+D_{F} \nu(t)
\end{aligned}
$$


satisfying

$$
[G(\lambda) F(\lambda)]=C(\lambda E-A)^{-1}\left[B_{G} B_{F}\right]+\left[D_{G} D_{F}\right]
$$

According to the system type, $\lambda$ also represents here either the differential operator $\lambda x(t)=\dot{x}(t)$ in the case of a continuous-time system or the advance operator $\lambda x(t)=x(t+1)$ in the case of a discrete-time system. Note that for most of practical applications $[G(\lambda) F(\lambda)]$ is a proper TFM, thus we can always choose a realization such that $E=I$. However, for the sake of generality, we only assume that the pencil $A-\lambda E$ is regular, without assuming $E$ is nonsingular. In this way, we will also cover the most general case of solving rational linear systems.

Let $S_{G}(\lambda)$ and $S_{F}(\lambda)$ be the system matrix pencils associated to the realizations of $G(\lambda)$ and $F(\lambda)$

$$
S_{G}(\lambda)=\left[\begin{array}{c|c}
A-\lambda E & B_{G} \\
\hline C & D_{G}
\end{array}\right], \quad S_{F}(\lambda)=\left[\begin{array}{c|c}
A-\lambda E & B_{F} \\
\hline C & D_{F}
\end{array}\right]
$$

Using the straightforward relations

$$
\begin{aligned}
& {\left[\begin{array}{cc}
A-\lambda E & B_{G} \\
O & G(\lambda)
\end{array}\right]=\left[\begin{array}{cc}
I_{n} & O \\
-C(A-\lambda E)^{-1} & I_{p}
\end{array}\right] S_{G}(\lambda)} \\
& {\left[\begin{array}{cc}
A-\lambda E & B_{F} \\
O & F(\lambda)
\end{array}\right]=\left[\begin{array}{cc}
I_{n} & O \\
-C(A-\lambda E)^{-1} & I_{p}
\end{array}\right] S_{F}(\lambda)}
\end{aligned}
$$

it is easy to see that $X(\lambda)$ is a solution of $G(\lambda) X(\lambda)=F(\lambda)$ if and only if

$$
Y(\lambda)=\left[\begin{array}{cc}
Y_{11}(\lambda) & Y_{12}(\lambda) \\
Y_{21}(\lambda) & X(\lambda)
\end{array}\right]
$$

satisfies

$$
S_{G}(\lambda) Y(\lambda)=S_{F}(\lambda)
$$

The existence of the solution of (8) is guaranteed by (5), which is equivalent to

$$
\operatorname{rank} S_{G}(\lambda)=\operatorname{rank}\left[S_{G}(\lambda) S_{F}(\lambda)\right]
$$

It follows that instead of solving the rational equation $G(\lambda) X(\lambda)=F(\lambda)$, we can solve the polynomial equation (8) and take

$$
X(\lambda)=\left[\begin{array}{ll}
O & I_{m}
\end{array}\right] Y(\lambda)\left[\begin{array}{c}
O \\
I_{q}
\end{array}\right]
$$


In fact, since we are interested in the second block column $Y_{2}(\lambda)$ of $Y(\lambda)$, we need only to solve

$$
\left[\begin{array}{cc}
A-\lambda E & B_{G} \\
C & D_{G}
\end{array}\right] Y_{2}(\lambda)=\left[\begin{array}{c}
B_{F} \\
D_{F}
\end{array}\right]
$$

and compute $X(\lambda)$ as

$$
X(\lambda)=\left[\begin{array}{ll}
O & I_{m}
\end{array}\right] Y_{2}(\lambda)
$$

The condition (9) for the existence of a solution becomes

$$
\operatorname{rank}\left[\begin{array}{cc}
A-\lambda E & B_{G} \\
C & D_{G}
\end{array}\right]=\operatorname{rank}\left[\begin{array}{ccc}
A-\lambda E & B_{G} & B_{F} \\
C & D_{G} & D_{F}
\end{array}\right]
$$

To solve (10), we isolate a full rank part of $S_{G}(\lambda)$ by reducing it to a particular Kronecker-like form. Let $Q$ and $Z$ be orthogonal matrices to reduce $S_{G}(\lambda)$ to the Kronecker-like form

$$
\bar{S}_{G}(\lambda):=Q S_{G}(\lambda) Z=\left[\begin{array}{cccc}
B_{r} & A_{r}-\lambda E_{r} & A_{r, r e g}-\lambda E_{r, r e g} & * \\
0 & 0 & A_{r e g}-\lambda E_{r e g} & * \\
0 & 0 & 0 & A_{l}-\lambda E_{l}
\end{array}\right]
$$

where $A_{r e g}-\lambda E_{r e g}$ is a regular subpencil, the pair $\left(A_{r}-\lambda E_{r}, B_{r}\right)$ is controllable with $E_{r}$ nonsingular and the subpencil $A_{l}-\lambda E_{l}$ has full column rank. The above reduction can be computed by employing numerically stable algorithms as those proposed in (Varga, 1996; Beelen and Van Dooren, 1988).

If $\bar{Y}_{2}(\lambda)$ is a solution of the reduced equation

$$
\bar{S}_{G}(\lambda) \bar{Y}_{2}(\lambda)=Q\left[\begin{array}{c}
B_{F} \\
D_{F}
\end{array}\right]
$$

then $Y_{2}(\lambda)=Z \bar{Y}_{2}(\lambda)$ and thus

$$
X(\lambda)=\left[\begin{array}{ll}
O & I_{m}
\end{array}\right] Z \bar{Y}_{2}(\lambda)
$$

is a solution of the equation $G(\lambda) X(\lambda)=F(\lambda)$. Partition

$$
Q\left[\begin{array}{l}
-B_{F} \\
-D_{F}
\end{array}\right]=\left[\begin{array}{l}
\bar{B}_{1} \\
\bar{B}_{2} \\
\bar{B}_{3}
\end{array}\right]
$$


in accordance with the row structure of $\bar{S}_{G}(\lambda)$. Since $A_{l}-\lambda E_{l}$ has full column rank, it follows from (11) that $\bar{B}_{3}=0$. Thus, we can choose $\bar{Y}_{2}(\lambda)$ of the form

$$
\bar{Y}_{2}(\lambda)=\left[\begin{array}{c}
\bar{Y}_{12}(\lambda) \\
\bar{Y}_{22}(\lambda) \\
\bar{Y}_{32}(\lambda) \\
O
\end{array}\right],
$$

where the partitioning of $\bar{Y}_{2}(\lambda)$ corresponds to the column partitioning of $\bar{S}_{G}(\lambda)$. Choosing $\bar{Y}_{12}(\lambda)=0$, we obtain

$$
\left[\begin{array}{l}
\bar{Y}_{22}(\lambda) \\
\bar{Y}_{32}(\lambda)
\end{array}\right]=\left[\begin{array}{cc}
\lambda E_{r}-A_{r} & \lambda E_{r, r e g}-A_{r, r e g} \\
O & \lambda E_{r e g}-A_{r e g}
\end{array}\right]^{-1}\left[\begin{array}{c}
\bar{B}_{1} \\
\bar{B}_{2}
\end{array}\right]
$$

Let partition $\left[\begin{array}{ll}O & I_{m}\end{array}\right] Z$ in accordance with the column structure of $S_{G}(\lambda)$ as

$$
\left[\begin{array}{ll}
O & I_{m}
\end{array}\right] Z=\left[D_{r} C_{r} C_{r e g} C_{l}\right]
$$

and denote

$$
\bar{A}-\lambda \bar{E}=\left[\begin{array}{cc}
A_{r}-\lambda E_{r} & A_{r, r e g}-\lambda E_{r, r e g} \\
O & A_{r e g}-\lambda E_{r e g}
\end{array}\right], \quad \bar{B}=\left[\begin{array}{c}
\bar{B}_{1} \\
\bar{B}_{2}
\end{array}\right], \quad \bar{C}=\left[C_{r} C_{r e g}\right]
$$

Then a particular solution $X_{0}(\lambda)$ of the equation $G(\lambda) X(\lambda)=F(\lambda)$ can be expressed in form of a descriptor realization

$$
X_{0}(\lambda)=\bar{C}(\lambda \bar{E}-\bar{A})^{-1} \bar{B}
$$

To compute $X_{0}(\lambda)$ we employed exclusively orthogonal similarity transformations. Therefore, this step is numerically stable, because we can easily show that the computed system matrices in the presence of roundoff errors are exact for an original problem with slightly perturbed data.

Some properties of $X_{0}(\lambda)$ can be easily deduced from the computed Kronecker-like form. The poles of $X_{0}(\lambda)$ are among the generalized eigenvalues of the pair $(\bar{A}, \bar{E})$ and are partly fixed, and partly freely assignable. The fixed poles represent the controllable eigenvalues of the pair $\left(\bar{B}_{2}, A_{r e g}-\lambda E_{r e g}\right)$. The generalized eigenvalues of the pair $\left(A_{r}, E_{r}\right)$ are called the "spurious" poles, and they originate from the column singularity of $G(\lambda)$. These poles are in fact freely assignable by appropriate choice of a (non-orthogonal) right transformation matrix (Varga, 2002).

If $G(\lambda)$ and $F(\lambda)$ have no common zeros then the pair $\left(\bar{B}_{2}, A_{r e g}-\lambda E_{r e g}\right)$ is controllable. This condition is always fulfilled in the case of solving a system (4) originating 
from FDI problems, where $F(\lambda)=\left[\begin{array}{ll}I & 0\end{array}\right]^{T}$ is a constant full column rank matrix. In this case, the solution $X_{0}(\lambda)$ will be proper if $\left[G_{f}(\lambda) G_{d}(\lambda)\right]$ (see (1) ) has no infinite zeros (i.e., all infinite eigenvalues of the matrix pair $\left(A_{\text {reg }}, E_{\text {reg }}\right)$ are simple). Moreover, a stable and proper solution will exist provided $\left[G_{f}(\lambda) G_{d}(\lambda)\right]$ is additionally minimum-phase.

More generally, the solution $X(\lambda)$ of $G(\lambda) X(\lambda)=F(\lambda)$ will have no pole in $\gamma$ (finite or infinite) if $c_{\gamma}(G)=c_{\gamma}([G F])$, where $c_{\gamma}(G)$ is the content of $G(\lambda)$ in $\gamma$ as defined by Verghese and Kailath (1981). Roughly, this is equivalent to say that the pole and zero structures of $G(\lambda)$ and $[G(\lambda) F(\lambda)]$ at $\gamma$ coincide. For practical computations, this implies that some or all of common zeros of $G(\lambda)$ and $[G(\lambda) F(\lambda)]$ will cancel. This cancellation can be done either explicitly by removing the uncontrollable eigenvalues of the pair $\left(\bar{B}_{2}, A_{\text {reg }}-\lambda E_{\text {reg }}\right)$ using orthogonal staircase algorithms (Varga, 1990), or implicitly at the next steps, during determining $\widehat{X}_{0}(\lambda)$ and $\widehat{X}(\lambda)$.

Remark. In this moment, we can easily determine a stable and proper solution of (4) by choosing an invertible diagonal $M(\lambda)$ such that $X(\lambda):=X_{0}(\lambda) M(\lambda)$ is stable and proper. The computation of $M(\lambda)$ can be done using methods discussed in (Varga, 2002). Note however that the resulting solution is usually not of least McMillan degree. Therefore, the next steps of the proposed procedure address exclusively the least order aspect.

\subsection{Computation of $X_{N}(\lambda)$}

Using the same reduction of $S_{G}(\lambda)$ to $\bar{S}_{G}(\lambda)$ as in (12), a nullspace basis $X_{N}(\lambda)$ of $G(\lambda)$ can be computed from a nullspace basis $\bar{Y}_{N}(\lambda)$ of $\bar{S}_{G}(\lambda)$ as

$$
X_{N}(\lambda)=\left[\begin{array}{ll}
O & I_{m}
\end{array}\right] Z \bar{Y}_{N}(\lambda)
$$

We can determine $\bar{Y}_{N}(\lambda)$ in the form

$$
\bar{Y}_{N}(\lambda)=\left[\begin{array}{c}
I \\
\left(\lambda E_{r}-A_{r}\right)^{-1} B_{r} \\
O \\
O
\end{array}\right] .
$$

With $C_{r}$ and $D_{r}$ defined in (14), we obtain a descriptor realization of $X_{N}(\lambda)$ as

$$
X_{N}(\lambda)=C_{r}\left(\lambda E_{r}-A_{r}\right)^{-1} B_{r}+D_{r} .
$$

Note that $X_{N}(\lambda)$ is a proper TFM which has least McMillan degree (Varga, 2003a). Moreover, the poles of $X_{N}(\lambda)$ are freely assignable by appropriately choosing the transformation matrices $Q$ and $Z$ to reduce the system pencil $S_{G}(\lambda)$. 
Remark. We can express $X_{N}(\lambda)$ to have the same state, descriptor and output matrices as $X_{0}(\lambda)$. If we denote $\bar{B}_{r}=\left[\begin{array}{c}B_{r} \\ O\end{array}\right]$, then $X_{N}(\lambda)$ can be also expressed as

$$
X_{N}(\lambda)=\bar{C}(\lambda \bar{E}-\bar{A})^{-1} \bar{B}_{r}+D_{r}
$$

This representation is evidently not minimal, since all generalized eigenvalues of the pair $\left(A_{\text {reg }}, E_{\text {reg }}\right)$ are uncontrollable.

\subsection{Selecting $M_{f}(\lambda)$}

In principle, this computation can be done simply by solving $q$ independent proper right coprime factorization $(\mathrm{RCF})$ problems for the single-input systems corresponding to each of $q$ columns of $X_{0}(\lambda)$. Assuming $X_{0, i}(\lambda)$ is the $i$-th column of $X_{0}(\lambda)$, we can compute the proper RCF

$$
X_{0, i}(\lambda)=\frac{\widehat{X}_{0, i}(\lambda)}{m_{f, i}(\lambda)}
$$

where $m_{f, i}(\lambda)$ and $\widehat{X}_{0, i}$ are both proper and mutually coprime. The resulting scalar transfer-function $m_{f, i}(\lambda)$ and rational vector $\widehat{X}_{0, i}(\lambda)$ are the $i$-th diagonal element of $M_{f}(\lambda)$ and the $i$-th column of $\widehat{X}_{0}(\lambda)$, respectively.

The transfer functions $m_{f, i}(\lambda)$ can be chosen, for example, in the form

$$
m_{f, i}(\lambda)=\frac{1}{(\lambda+\alpha)^{n_{\infty}, i}},
$$

where $n_{\infty, i}$ is the number of infinity zeros of $X_{0, i}(\lambda)$ and $\alpha$ is an arbitrary value, representing a desired stability degree for the solution. It is possible to determine $n_{\infty, i}$ efficiently from the resulting Kronecker-like form of the system pencil $S_{G}(\lambda)$. We can assume that $A_{r e g}-\lambda E_{r e g}$ and $\bar{B}_{2}$ are partitioned conformably and have the structure

$$
A_{r e g}-\lambda E_{r e g}=\left[\begin{array}{cc}
A_{f}-\lambda E_{f} & A_{f, \infty}-\lambda E_{f, \infty} \\
O & A_{\infty}-\lambda E_{\infty}
\end{array}\right], \quad \bar{B}_{2}=\left[\begin{array}{c}
\bar{B}_{f} \\
\bar{B}_{\infty}
\end{array}\right]
$$

where $A_{f}-\lambda E_{f}$ and $A_{\infty}-\lambda E_{\infty}$ contain the finite and infinite invariant zeros of $S_{G}(\lambda)$. If we denote $\bar{b}_{\infty, i}$ the $i$-th column of $\bar{B}_{\infty}$, then $n_{\infty, i}+1$ is just the order of the controllable part of the pair $\left(A_{\infty}-\lambda E_{\infty}, \bar{b}_{\infty, i}\right)$. To compute $n_{\infty, i}$ we can apply the generalized controllability staircase algorithm of Varga (1990) to the pair ( $\left.E_{\infty}-\lambda A_{\infty}, \bar{b}_{\infty, i}\right)$ (note that $A_{\infty}$ and $E_{\infty}$ are interchanged). 
After having determined a minimal state-space realization $\left(A_{M_{f}}-\lambda I, B_{M_{f}}, C_{M_{f}}, 0\right)$ for $M_{f}(\lambda)$, it is necessary to compute a proper descriptor representation of $\widehat{X}_{0}(\lambda)=$ $X_{0}(\lambda) M_{f}(\lambda)$. This can be done in two steps: first, remove all uncontrollable infinite eigenvalues from the state-space realization of $X_{0}(\lambda) M_{f}(\lambda)$ applying the algorithm of Varga (1990) and then remove the non-dynamic part applying standard techniques (Verghese et al., 1981). Both steps can be performed efficiently by exploiting the inherited structure of the system matrices of $X_{0}(\lambda)$ from the Kronecker-like structure of $S_{G}(\lambda)$. Note that in the first step we also eliminate the uncontrollable infinite eigenvalues originating from the common infinite poles and zeros of $G(\lambda)$ and $F(\lambda)$. We omit further details here because of lack of space.

\subsection{Computation of a least order $\widehat{X}(\lambda)$}

We assume that $\widehat{X}_{0}(\lambda)$ and $X_{N}(\lambda)$ are proper TFMs and possess state-space representations sharing the same descriptor, state and output matrices

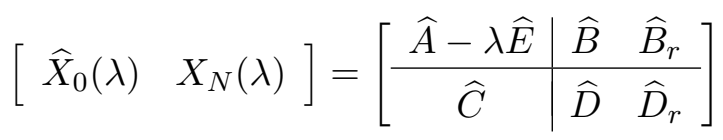

with $\widehat{E}$ non-singular. This can be easily achieved by performing all relevant transformations employed to eliminate the non-proper part of $X_{0}(\lambda) M_{f}(\lambda)$ also on the non-minimal realization (15) of $X_{N}(\lambda)$.

It was shown by Morse (1976) that computing a least order solution $\widehat{X}(\lambda)=\widehat{X}_{0}(\lambda)+$ $X_{N}(\lambda) Y(\lambda)$ by choosing an appropriate proper $Y(\lambda)$ is equivalent to determine a feedback matrix $\widehat{F}_{r}$ and a feedforward matrix $\widehat{L}_{r}$ to cancel the maximum number of unobservable and uncontrollable poles of

$$
\widehat{X}(\lambda)=\left[\begin{array}{c|c}
\widehat{A}+\widehat{B}_{r} \widehat{F}_{r}-\lambda \widehat{E} & \widehat{B}+\widehat{B}_{r} \widehat{L}_{r} \\
\hline \widehat{C}+\widehat{D}_{r} \widehat{F}_{r} & \widehat{D}+\widehat{D}_{r} \widehat{L}_{r}
\end{array}\right]
$$

It can be shown that if we start with a minimal realization of $[G(\lambda) F(\lambda)]$, then we can not produce any unobservable poles in $\widehat{X}(\lambda)$ via state-feedback. Therefore, we only need to determine the matrices $\widehat{F}_{r}$ and $\widehat{L}_{r}$ to cancel the maximum number of uncontrollable poles.

Morse (1976) has shown that this problem can be solved as a minimal order dynamic cover design problem. Consider the set

$$
\mathcal{J}=\left\{\mathcal{V}: \operatorname{Im} \bar{B}+\bar{A} \mathcal{V} \subset \operatorname{Im} \bar{B}_{r}+\mathcal{V}\right\}
$$


where $\bar{A}:=\widehat{E}^{-1} \widehat{A}, \bar{B}:=\widehat{E}^{-1} \widehat{B}$, and $\bar{B}_{r}:=\widehat{E}^{-1} \widehat{B}_{r}$. Let $\mathcal{J}^{*}$ denote the set of subspaces in $\mathcal{J}$ of least dimension. If $\mathcal{V} \in \mathcal{J}^{*}$, then a pair $\left(\widehat{F}_{r}, \widehat{L}_{r}\right)$ can be determined such that

$$
\left(\bar{A}+\bar{B}_{r} \widehat{F}_{r}\right) \mathcal{V}+\operatorname{Im}\left(\bar{B}+\bar{B}_{r} \widehat{L}_{r}\right) \subset \mathcal{V}
$$

Thus, determining a minimal dimension $\mathcal{V}$ is equivalent to a minimal order cover design problem, and a conceptual approach to solve it has been indicated by Morse (1976). The outcome of his method is, besides $\mathcal{V}$, the pair $\left(\widehat{F}_{r}, \widehat{L}_{r}\right)$ which achieves a maximal order reduction by forcing pole-zero cancellations. This approach has been turned into a numerically reliable procedure by Varga (2003b). In this procedure $\widehat{F}_{r}$ and $\widehat{L}_{r}$ are determined from a special controllability staircase form of the pair $\left(\widehat{A}-\lambda \widehat{E},\left[\widehat{B}_{r} \widehat{B}\right]\right)$ obtained by using a numerically reliable method relying on both orthogonal and non-orthogonal similarity transformations. An additional feature of this procedure is that all uncontrollable eigenvalues of the pair $(\widehat{A}-\lambda \widehat{E}, \widehat{B})$, arising from common poles or zeros of $G(\lambda)$ and $F(\lambda)$ are also eliminated.

\subsection{Selecting $M_{s}(\lambda)$}

The computation of $M_{s}(\lambda)$ can be done simply by solving $q$ stable RCF problems for the single-input systems corresponding to each column $\widehat{X}_{i}(\lambda)$ of $\widehat{X}(\lambda)$

$$
\widehat{X}_{i}(\lambda)=\frac{X_{i}(\lambda)}{m_{s, i}(\lambda)}
$$

One distinctive feature of these single-input factorization problems is that each $\widehat{X}_{i}(\lambda)$, has generally an uncontrollable descriptor realization. This aspect is handled automatically when employing the Algorithm GRCF-P of (Varga, 1998). Since each of resulting $m_{s, i}(\lambda)$ has least McMillan degree, the resulting diagonal matrix $M_{s}(\lambda)$ has least McMillan degree as well.

\section{EXAMPLES}

Example 1. Consider the following simple continuous-time example taken from (Frisk, 2000):

$$
G_{p}(s)=\left[\begin{array}{c}
\frac{1}{s+1} \\
\frac{1}{(s+1)^{2}}
\end{array}\right], G_{f}(s)=\left[\begin{array}{l}
0 \\
1
\end{array}\right], G_{d}=0
$$


A minimal order left-inverse of $G_{f}(s)$ is $Q(s)=\left[\begin{array}{ll}0 & 1\end{array}\right]$, which is proper and stable. According to Ding and Frank (1990), a residual generator can be determined in the observer-like form

$$
\mathbf{r}(s)=Q(s)\left(\mathbf{y}(s)-G_{p}(s) \mathbf{u}(s)\right) .
$$

This leads to a second order stable and proper detector

$$
R(s)=Q(s)\left[I-G_{p}(s)\right]=\left[\begin{array}{lll}
0 & 1 & -\frac{1}{(s+1)^{2}}
\end{array}\right]
$$

which is however not of least possible order.

We apply now the proposed approach to compute a least order detector. For this simple model we will explicitly manipulate rational matrices instead of state space matrices. The TFMs defining the equation (4) are given by

$$
G(s)=\left[\begin{array}{ccc}
0 & 1 & 0 \\
\frac{1}{s+1} & \frac{1}{(s+1)^{2}} & 1
\end{array}\right], \quad F=\left[\begin{array}{l}
1 \\
0
\end{array}\right]
$$

A particular solution $X_{0}(s)$ of the equation $G(s) X(s)=F$ and a rational nullspace basis $X_{N}(s)$ of $G(s)$ are

$$
X_{0}(s)=\left[\begin{array}{c}
0 \\
1 \\
-\frac{1}{(s+1)^{2}}
\end{array}\right], \quad X_{N}(s)=\left[\begin{array}{c}
-1 \\
0 \\
1 \\
\frac{1}{s+1}
\end{array}\right]
$$

Note that $X_{0}^{T}(s)$ is the second order detector determined previously. If we choose $Y(s)=\frac{1}{s+1}$, then we obtain a first order stable and proper detector

$$
R(s)=\left(X_{0}(s)+X_{N}(s) Y(s)\right)^{T}=\left[\begin{array}{lll}
-\frac{1}{s+1} & 1 & 0
\end{array}\right]
$$

having the least possible McMillan degree.

Example 2. This example is the descriptor system described in (Hou, 2000) corresponding to a linearized three-links planar manipulator model. This model has state vector dimension $n=11$, command input vector dimension $m=3$, fault vector dimension $q=2$, no disturbance input, and output vector dimension $p=4$. This model is not minimal and a minimal realization has order 5 and is proper.

The method proposed by Hou (2000) is essentially equivalent to design two independent FDI filters. By considering the fault input 1 as fault and fault input 2 as 
disturbance, a 4-th order FDI filter $R_{1}(s)$ has been designed by Hou (2000). Similarly, by considering fault input 2 as fault and fault input 1 as disturbance, he obtained a 4-th order FDI filter $R_{2}(s)$. In this way, a FDI filter of order 8 has been determined by stacking the two designed filters

$$
R(s)=\left[\begin{array}{l}
R_{1}(s) \\
R_{2}(s)
\end{array}\right]
$$

By using the new approach proposed in this paper we can determine a residual generator which has a least order equal to 2 . In the rational system (4) to be solved $G(s)$ is $5 \times 7$ and $F(s)$ is $5 \times 2$, thus $R(s)=X^{T}(s)$ will be a $2 \times 7$ matrix. A particular solution $X_{0}(s)$ has been determined having a state space realization of order 10, with the pair $(\bar{A}, \bar{E})$ having 3 finite and 7 infinite generalized eigenvalues. The nullspace basis $X_{N}(s)$ is $7 \times 2$ and has dynamical order 3 . With $M_{f}(s)$ of the form $M_{f}(s)=\frac{1}{s+1} I_{2}$, we can eliminate all infinite poles of $X_{0}(s)$ and the resulting proper solution $\widehat{X}_{0}(s)=X_{0}(s) M_{f}(s)$ has order 5. After performing the minimum cover design, we get a stable solution $X(s)=\widehat{X}(s)$ of order 2 with both eigenvalues stable and equal to -1. For reference purposes we give the resulting 2-nd order FDI filter

$$
R(s)=\left[\begin{array}{ccccc}
-\frac{0.01042 s+0.04455}{s+1} & \frac{0.03462}{s+1} & -\frac{0.03899 s+1.936}{s+1} \\
\frac{1}{s+1} & \frac{s}{s+1} & 0 & \\
\ldots & -\frac{0.02753 s+1.377}{s+1} & 0 & \frac{0.03899}{s+1} & \frac{0.02753}{s+1} \\
0 & -\frac{1}{s+1} & 0 & 0
\end{array}\right]
$$

\section{CONCLUSIONS}

We proposed numerically reliable approaches to solve several basic computational problems encountered in the design of FDI filters, namely: (1) the solution of linear rational equations; (2) the computation of rational nullspace bases of rational matrices; (3) the reduction of the dynamical orders of the solutions by employing minimal dynamic cover design techniques; and (4) the computation of stable and proper rational factorizations with diagonal denominators. Each of these computations can be performed using numerically stable or numerically reliable algorithms. Using such algorithms, the FDI problem can be solved in the most general setting. Our approach provides, for the first time, a satisfactory numerical solution to this problem. Note 
that least order residual generator design algorithms have been already proposed to solve the simpler fault detection problem (i.e., without isolation) by Frisk and Nyberg (2001) using a polynomial basis approach, and by Varga (2003a) using state space computational techniques.

For the implementation of the proposed residual generator design approach, all necessary basic numerical software is available in the DESCRIPTOR SYSTEMS Toolbox for MATLAB (Varga, 2000), as for example, the computation of Kronecker-like staircase forms, computation of standard and special controllability forms (required in minimum cover design), computation of poles and zeros of descriptor systems, determination of minimal realizations, stable coprime factorization, etc. The basic computational tools in this toolbox are several functionally rich $M E X$-functions, representing MATLAB interfaces to powerful and numerically robust Fortran subroutines partly available in the control and systems library SLICOT (Benner et al., 1999).

\section{REFERENCES}

Beelen, T. and P. Van Dooren (1988). An improved algorithm for the computation of Kronecker's canonical form of a singular pencil. Lin. Alg. \& Appl. 105, 9-65.

Benner, P., V. Mehrmann, V. Sima, S. Van Huffel and A. Varga (1999). SLICOT a subroutine library in systems and control theory. In: Applied and Computational Control, Signals and Circuits (B. N. Datta, Ed.), Vol. 1., pp. 499-539. Birkhäuser.

Chen, J. and R. J. Patton (1999). Robust Model-Based Fault Diagnosis for Dynamic Systems, Kluwer Academic Publishers, London.

Ding, X. and P. M. Frank (1990). Fault detection via factorization. Systems \& Control Lett. 14, 431-436.

Frank, P. M. and X. Ding (1994). Frequency domain approach to optimally robust residual generation and evaluation for model-based fault diagnosis. Automatica 30, 789-804.

Frisk, E. (2000). Order of residual generators - bounds and algorithms. Prepr. IFAC Symp. SAFEPROCESS'2000, Budapest, Hungary, pp. 599-604.

Frisk, E. and M. Nyberg (2001). A minimal polynomial basis solution to residual generation for fault diagnosis in linear systems. Automatica 37, 1417-1424.

Gertler, J. (1998). Fault Detection and Diagnosis in Engineering Systems, Marcel Dekker, New York. 
Gertler, J. (2000). Designing dynamic consistency relation for fault detection and isolation. Int. J. Control 73, 720-732.

Gertler, J. J. and R. Monajemy (1995). Generating directional residuals with dynamic parity relations. Automatica 31, 627-635.

Hou, M. (2000). Fault detection and isolation for descriptor systems. In: Issues of Fault Dyagnosis for Dynamic Systems (R. J. Patton, P. M. Frank and R. N. Clark, Eds.), Springer Verlag, London. pp. 115-144.

Morse, A. S. (1976). Minimal solutions to transfer matrix equations. IEEE Trans. Autom. Control 21, 131-133.

Peng, Y., A. Youssouf, Ph. Arte and M. Kinnaert (1997). A complete procedure for residual generation and evaluation with application to a heat exchanger. IEEE Trans. Control Systems Technology 5, 542-555.

Varga, A. (1990). Computation of irreducible generalized state-space realizations. Kybernetika 26, 89-106.

Varga, A. (1996). Computation of Kronecker-like forms of a system pencil: Applications, algorithms and software. Proc. CACSD'96 Symposium, Dearborn, MI, pp. 77-82.

Varga, A. (1998). Computation of coprime factorizations of rational matrices. Lin. Alg. \& Appl. 271, 83-115.

Varga, A. (2000). A descriptor systems toolbox for MATLAB. Proc. CACSD’2000 Symposium, Anchorage, Alaska.

Varga, A. (2002). Computational issues in fault-detection filter design. Proc. of CDC'2002, Las Vegas, Nevada.

Varga, A. (2003a). On computing least order fault detectors using rational nullspace bases. Prepr. of IFAC Symp. SAFEPROCESS'2003, Washington D.C.

Varga, A. (2003b). Reliable algorithms for computing minimal dynamic covers. (submitted to CDC'2003).

Verghese, G. and T. Kailath (1981). Rational matrix structure. IEEE Trans. Autom. Control 26, 434-439.

Verghese, G., B. Lévy and T. Kailath (1981). A generalized state-space for singular systems. IEEE Trans. Autom. Control 26, 811-831.

Wang, S.-H. and E. J. Davison (1973). A minimization algorithm for the design of linear multivariable systems. IEEE Trans. Autom. Control 18, 220-225. 